\title{
L'alimentation et le sourire : la communication corporative chez Danone
}

Maria MERINO

\section{(2) OpenEdition \\ Journals}

Édition électronique

URL : http://journals.openedition.org/transtexts/621

DOI : $10.4000 /$ transtexts.621

ISSN : 2105-2549

Éditeur

Gregory B. Lee

Référence électronique

Maria MERINO, "L'alimentation et le sourire : la communication corporative chez Danone »,

Transtext(e)s Transcultures 跨文本跨文化 [En ligne], 10 | 2015, mis en ligne le 14 juillet 2016, consulté le 10 décembre 2020. URL : http://journals.openedition.org/transtexts/621 ; DOI : https://doi.org/ $10.4000 /$ transtexts.621

Ce document a été généré automatiquement le 10 décembre 2020.

(c) Tous droits réservés 


\title{
L'alimentation et le sourire : la communication corporative chez Danone
}

\author{
Maria MERINO
}

\section{L'alimentation et le sourire : la communication corporative chez Danone}

1 En plein contexte de crise économique qui frappe l'Europe et plus particulièrement l'Espagne, Danone a lancé en avril 2013, une nouvelle campagne publicitaire « Danone, alimente le sourire ». Il n'y a aucun doute sur le message véhiculé par cette campagne : son but est de donner espoir dans un contexte de crise. Le public ciblé n'est pas le client en tant qu'individu propre mais aux familles en tant que principale unité de consommation et au sein de laquelle se tissent les relations affectueuses.

2 Tout au long de son histoire, Danone a été au coté des familles en leur offrant des produits alimentaires de qualité. La nouvelle campagne publicitaire présente une différence majeure qui réside dans le fait qu'en aucune occasion n'apparaît le mot santé et que le ton des messages publicitaires ne correspond pas au domaine de la publicité, c'est-à-dire de la persuasion, mais plutôt à celui des relations publiques, dont le but est de créer des liens et des relations entre une organisation ou une marque et ses clients. Cette démarche passe particulièrement par l'utilisation du langage des émotions. Afin de mieux comprendre l'apparition de cette nouvelle campagne, il est tout d'abord nécessaire d'étudier succinctement l'histoire du produit et la communication de celui-ci au travers de la publicité. 


\section{Les marques de fabrique de Danone}

3 Même si la marque Danone n'a pas besoin d'être présentée, il est cependant nécessaire de souligner quelques aspects liés á son origine et qui constituent les marques de fabrique de l'entreprise tout au long de son histoire.

4 C'est la famille Carasso qui est à l'origine de Danone. Il s'agit d'une famille juive, séfarade, installée à Thessalonique en Grèce depuis leur expulsion d'Espagne en 1492. Cependant la famille n'a jamais renoncé à ses origines espagnoles, c'est pourquoi, quand quatre cents ans plus tard, aux prémisses de la Première Guerre mondiale la ville de Thessalonique se retrouve dans une situation instable, un des descendants, Isaac, retourna en Espagne où il s'installa à Barcelone. Sur place il se rendit compte que les enfants souffraient de nombreux problèmes intestinaux et digestifs. Isaac connaissait les découvertes du Prix Nobel russe Ilya Ilyich Mechnikov, fondateur de la science de l'immunologie qui défendait les bénéfices du lait fermenté pour la santé. Dans les Balkans la consommation de ce produit était habituelle et le professeur affirmait que la longévité de ces peuples était en partie due à la grande consommation de ce type d'aliments. En Europe occidentale le yaourt n'existait pas encore mais Isaac Carasso connaissait son existence de par ses voyages en Bulgarie où ceux-ci étaient vendus au litre dans des étals par des vendeurs de rue. Isaac décida donc de commencer la fabrication et la commercialisation de yaourt en Espagne. C'est ainsi qu'en 1919 naquit le premier yaourt Danone, nom qui tire son origine du diminutif de Daniel le fils d'Isaac, qui plus tard, reprendra les rennes de l'entreprise.

5 Durant les premières années, les yaourts se vendaient exclusivement en pharmacie, de ce fait, en plus des propriétés intrinsèques du produit liées à la santé, celui-ci bénéficia de la garantie de qualité apportée par la vente en pharmacie et la prescription médicale. Danone et la santé étaient liées à vie et ceci constitue d'ailleurs une de ses marques de fabrique. La deuxième marque de fabrique se manifeste par l'innovation issue de la personnalité de pionner du créateur Isaac Carasso. Enfin la troisième marque de fabrique réside dans la recherche, intimement liée à la qualité, qui fut présente des les débuts de l'entreprise quant Isaac envoya son fils Daniel faire des études de commerce à Marseille et étudier la bactériologie à l'Institut Pasteur à Paris. C'est dans cette ville que Daniel commencera la fabrication des produits Danone en 1929.

6 Ces quelques éléments sur les débuts de l'aventure de l'entreprise d'Isaac Carasso et continuée par son fils Daniel, marquent le ton de la culture de l'entreprise : innovation, santé et qualité matérialisé dans des aliments qui aident à protéger la santé, et en premier lieu celle des enfants. C'est sur ces valeurs que s'est basée la publicité depuis les débuts de l'entreprise. Au fil des années le terme de santé s'est vu doté d'autres nuances : santé et sport, santé et bien-être physique. Le produit est aussi recommandé pour la santé des adultes.

\section{Faire connaître un produit alimentaire nouveau}

7 Lors de la commercialisation d'un nouveau produit le choix d'un nom joue un rôle important. La simplicité du terme Danone permet à la marque d'être facilement mémorisée tant en Espagne qu'en France, où l'entreprise s'est installée par la suite. 
8 «(..) Pour que les institutions puissent jouir d'un certain prestige il est nécessaire que le public reconnaissent leurs noms et qu'il se rappelle au moins vaguement pour quelle raison celles-ci méritent leur respect. Par ailleurs une institution renommée, véhicule plus de confiance, favorise l'identification collective et permet une plus grande diffusion des valeurs qu'elle incarne » Gerd Gigerenzer, Directeur du Centre sur le Comportement Adaptatif et la Cognition de l'Institut Max Planck pour le développement humain à Berlin.

9 L'un des problèmes auxquels est confrontée la publicité des produits alimentaires est de relier ces derniers au concept de santé. La santé est une valeur à laquelle le public est très sensible mais dans le même temps cette valeur varie selon l'évolution des tendances de la société. De nos jours certains produits contenant des ingrédients qui peuvent provoquer une prise de poids - graisses, glucides ou sucres -, ce qui reste un tabou dans notre perception actuelle du bien-être, sont véritablement diabolisés.

10 Cette situation ne s'applique pas à Danone puisque le produit depuis son origine est lié au concept de santé, ce que Carasso a bien su communiquer. Au début de son activité il a consacré une grande partie de ses efforts à informer les médecins sur les vertus du yaourt. Il entreprit même une campagne de distribution journalière d'échantillons aux membres du corps médical. Ses efforts portèrent rapidement leurs fruits, les médecins commencèrent à prescrire du yaourt à leurs patients qui à leur tour se dirigeaient dans les pharmacies afin de les acheter, celles-ci étant le seul endroit où ils étaient dispensés.

11 Les premières publicités mentionnaient déjà le terme de santé. Par exemple on peut citer le slogan que, dès 1930, R.L Dupuy imprima sur une affiche publicitaire en France «délicieux et sain, le yoghourt Danone est le dessert des digestions heureuses!» Le message publicitaire apporte la nuance suivante : Il ne s'agit pas d'un médicament mais il ne s'agit pas non plus d'un simple aliment mais d'un aliment sain qui peut être consommé comme un délicieux dessert. Ainsi la notion de santé reste liée à celle de plaisir.

12 Une autre des marques de fabrique de Danone a toujours été, comme nous l'avons déjà signalé, l'innovation. Danone est né des fruits de l'innovation qui lui a servi de guide tout au long de son existence faisant aussi de nécessité vertu. En effet, l'invasion nazie en France contraint la famille Carasso à émigrer aux Etats-Unis. Cet exil obligé fut l'occasion d'élargir l'entreprise familiale mais aussi de l'adapter au marché américain. Des parfums furent ajoutés aux yaourts, ce fut le premier pas d'une série d'innovations: yaourts écrémés, actimel, yaourts enrichis en propriétés renforçant la notion de santé, yaourts destinés à des publics spécifiques (yaourts pour garder la ligne, yaourts au soja pour les femmes, yaourts spéciaux pour les enfants, etc.) ainsi que de nouveaux produits. Ce mode d'action a conforté l'image de Danone comme une marque qui se développait et s'améliorait au même rythme qu'évoluaient les nécessités et les goûts des familles et vice versa. En effet les familles ont aussi modifié leurs habitudes alimentaires en fonction des changements qui se produisaient sur le marché.

Quelque temps plus tard, à la notion de santé s'ajoute celle du sport. La consommation de yaourt permet d'avoir un corps en forme. Danone sponsorise des évènements sportifs, de sorte que Danone et le sport soient automatiquement associés.

14 L'alliance de la santé et du sport a donné le jour à l'image des « corps Danone » : la beauté d'un corps jeune, musclé et bien dessiné est devenu l'icône de la bonne santé et de la beauté. Il s'agit de temps économiques prospères durant lesquels l'expression «corps 
Danone » passe dans le langage populaire pour définir une personne particulièrement attirante.

Jusqu'alors toutes les campagnes publicitaires se centraient sur les propriétés bénéfiques du yaourt. Cependant la configuration du marché et de la communication changent et les messages publicitaires se transforment jusqu'à arriver à cette dernière campagne publicitaire de laquelle la notion de santé est absente et où il est uniquement fait appel aux sentiments.

\section{La publicité dans un monde envahi par la communication}

Les spécialistes de la communication des entreprises $\operatorname{Costa}^{2}$ et Capriotti ${ }^{3}$ affirment que nous arrivons à la fin d'une époque dans laquelle les entreprises faisaient la promotion de leurs produits en se basant sur trois outils principaux: la propagande, la publicité à grande échelle et le marketing. Les raisons de ce changement sont dues à la nouvelle configuration du marché et de la communication. Nous nous trouvons face à un monde saturé de messages publicitaires et d'offres de produits similaires auquel nous sommes parvenu grâce au développement et à l'accès facile aux technologies de la communication, ce qui oblige les marques à adopter un autre discours plus profond que celui de la simple persuasion. La nécessité de se démarquer d'une si nombreuse concurrence a provoqué un glissement de la publicité du produit à la publicité de l'entreprise ou de la marque. C'est une image globale qui est recherchée, une image qui vante non seulement les bienfaits d'un produit mais aussi de celui qui le fabrique. L'entreprise doit non seulement montrer que les produits qu'elle commercialise sont bons mais qu'elle aussi est une bonne entreprise, elle doit répondre de son fonctionnement de ses différentes politiques, de gestion, politiques internes, commerciales, d'approvisionnement, de ses relations avec la société. Apparait le terme "d'image corporative " par lequel s'exprime l'idée que les différents publics, pas uniquement les clients, se forgent l'idée d'une marque, d'une entreprise ou d'une organisation. Réduite à sa plus simple expression cela peut être une image positive ou négative. Entre ces deux pôles se tissent les attitudes que le public adopte envers les entreprises.

$\mathrm{Au}$ sein de l'organisation de l'entreprise on voit se développer le concept de responsabilité sociale : l'entreprise doit rendre à la société en retour de ce que celle-ci lui met à disposition pour se constituer, une partie de ses bénéfices. Elle devient un sujet actif de la vie de la société et veille à ce que le jugement que celle-ci lui porte soit positif. Au-delà du marketing et de la publicité les entreprises entrent en relation avec l'environnement dans lequel elles évoluent et avec lequel elles interagissent.

18 A la situation du marché s'ajoute la révolution qu'a traversé le monde de la communication depuis l'apparition de l'internet, avec sa facilité à favoriser la mondialisation. Le web 2.0 a de nouveau révolutionné la communication en octroyant un rôle important aux récepteurs de communications qui peuvent désormais se convertir, à leur tour, en émetteur. C'est la fin de la communication à un sens. Il faut donc penser à comment gagner des batailles dans le nouveau modèle de communication à double sens. 


\section{Le rôle des émotions}

19 Dans le contexte d'un monde envahi par la communication et saturé d'offres, des études voient le jour sur la réception de la publicité. Entre en scène l'importance des émotions, élément qu'Aristote avait qualifié d'essentiel dans les relations humaines et la communication persuasive. Les premières études menées dans les années 50 se concentrent sur la persuasion ${ }^{4}$. Elles sont reprises par l'Ecole de Yale, desquels auteurs on peut citer J. Mc Guire ${ }^{5}$ qui proposa, en 1978, un important modèle de hiérarchie des effets publicitaires. Déjà dans les années 80 le débat tourne autour du rôle des émotions dans les processus d'information. Ces dernières sont reconnues comme des facteurs de premier ordre, selon Zajonc ${ }^{6}$ et Bower ${ }^{7}$. Les émotions amènent le récepteur, stimulé par des forces externes, à répondre de manière réactive. Ceci induit un changement par rapport au modèle antérieur qui plaçait au centre de son action le caractère actif du récepteur, qui était considéré maître de ses propres pensées, sensations et conduites ce qui justifiait le besoin de développer des techniques de persuasion. Ces observations commencent à s'appliquer au monde du marketing et de la publicité, Holbrook et Hirchsman ${ }^{8}$, tendance qui reste toujours d'actualité.

Les émotions peuvent être définies, au niveau neurologique, comme un processus chimique qui se produit dans le cerveau en réponse à une stimulation déterminée. D'où l'intérêt de susciter des émotions positives chez le client potentiel afin d'influer sur ce dernier pour qu'il agisse au-delà du rationnel, par empathie envers la marque ou l'entreprise.

21 «Créer un lien émotionnel avec le consommateur, qui choisit la marque parce qu'elle lui plait, parce qu'elle le comprend, au-delà de ce qu'elle vend. Il est prouvé qu'à l'heure de choisir un produit, si une marque s'adresse à moi d'une manière plus directe que les autres, qu'elle se relie à moi, qu'elle évoque des choses auxquelles je m'identifie, je la choisirais, bien qu'elle soit un peu plus chère. Il s'agit de créer une sorte de tendresse envers les marques » affirme Raquel Martinez, directrice de création de l'agence McCann Erickson. ${ }^{9}$

22 C'est ainsi que la communication des entreprises s'est tournée vers la diffusion de ces valeurs qui suscitent des sentiments positifs. Revenons à Danone et arrêtons-nous sur quelques une de leurs propositions.

23 En 2008 Danone commence la campagne de solidarité « Ensemble Semons la Vie » grâce à laquelle une part de chaque achat est destinée à la collecte de fonds pour l'achat d'un milliard de semences que la Croix Rouge destine à des projets de sécurité alimentaire en Afrique.

24 La publicité se concentre plus sur la communication des émotions. Le dernier exemple étant celui que nous étudions aujourd'hui « Danone, alimente le sourire ».

La campagne publicitaire a commencé en avril 2013, en Espagne, avec un spot télévisuel dans lequel un enfant s'approche du ventre de sa mère enceinte pour parler à son frère qui n'est pas encore né. Le dialogue est le suivant:

Salut, comment ça va la dedans? Ici tout le monde parle de la crise. Quand tu connaîtras papa et maman ils te paraîtront bizarres. Ils ne sont pas comme ça tout le temps, ils sont juste inquiets. Mais tu sais quoi? Moi j'ai envie que tu arrives. Et je veux que l'on joue 
ensemble. Et j'ai beaucoup de choses pour toi ...! Mes T-shirts, mes dinosaures ... mais mes danones ils sont à moi. Bon, un jour je te laisserai les goûter.

Le dialogue compte 65 mots, dans sa version originale espagnole, suffisants pour créer le climat désiré : intimiste, familier, pacifique, encourageant, optimiste, authentique. Le protagonisme de l'enfance est un symbole clair et efficace pour parler d'espérance. Celleci est magistralement personnifiée au travers d'une créature qui n'est pas encore née mais qui est déjà là et qui constitue le futur proche de la famille, un de ses membres avec lequel il est possible de parler, de rêver, de l'inviter à partager des jeux. La joie de la prochaine naissance qui aura bientôt lieu contraste avec le contexte pessimiste de crise économique, qui est simplement mentionné, mais dépassé par l'amour.

Les images sont accompagnées de la célèbre chanson des Beatles, Let It Be reprise par le groupe Vazquez Sounds, groupe musical formé par trois jeunes frères adolescents. Ce choix renforce les messages de confiance dans le futur et de soutien à la famille. Les jeunes, qui se basent sur la tradition culturelle des générations antérieures, la renouvelle en lui apportant une nouvelle vitalité et en récoltant de nouveau les fruits de son succès, comme c'est le cas du groupe mexicain.

Pendant que l'enfant s'adresse à son frère, les parents sont en train de se reposer tranquillement, peut-être sont-ils en train de faire la sieste après avoir mangé et fini le repas par un dessert Danone. Tous ces éléments renforcent le concept de famille, de spontanéité, d'authenticité, de paix, de tendresse, de simplicité et d'espérance. Le tout invite à esquisser un sourire. Quant celui-ci à été esquissé et que la publicité prend fin, six mots la concluent " Danone, nous sommes à vos côtés.». Le sourire représente le bonheur et Danone est assimilé à ce bonheur.

30 A cette communication émotionnelle s'ajoute toute une campagne que l'on nomme aujourd'hui communication à $360^{\circ}$, c'est-à-dire une communication qui inclut la publicité, les relations publiques ainsi que le marketing dans ses différentes stratégies. L'une d'entre elles explicite la rationalité qui se cache derrière l'émotion comme c'est le cas d'un autre spot audiovisuel dans lequel sont détaillés les effets du sourire :

31 «Comment te sens-tu aujourd'hui? Un jour de plus? Ou tu penses que cela va être un grand jour? De nombreux facteurs influent sur notre humeur. Mais il y a quelque chose, un geste tout simple capable de changer la journée : le sourire. Ce geste si commun cache d'incroyables pouvoirs. Il y a quelque chose de spécial dans un sourire, il n'est pas appris. Avant même de naître les bébés sourient dans le ventre de leur mère. Ce sont les enfants qui sourient le plus, jusqu'à 400 fois par jour. En revanche, les adultes sourient, tout au plus, 20 fois par jour. Quel dommage. Il a été démontré que le sourire, en plus de changer l'expression du visage, a aussi une influence sur nos émotions. C'est-à-dire que par simple fait de sourire nous nous sentons mieux. L'effet d'un sourire peut être, par exemple, comparable au plaisir procuré par la consommation de deux mille barres de chocolat. De plus, selon diverses études, il a été prouvé que les personnes qui sourient le plus sont celles qui vivent le plus longtemps.

Mais le meilleur de tout cela c'est que lorsque nous voyons un sourire nous l'imitons. Nous sourions 30 fois plus en groupe que seuls. C'est ainsi que lorsque nous sourions, non seulement nous nous sentons biens, mais en plus nous propageons notre bien-être aux personnes qui nous entourent. Une chaîne que l'on pourrait commencer chaque jour. Nous savons que nous ne pouvons pas changer le monde d'un jour à l'autre, mais chez Danone nous savons que nous tous nous pouvons améliorer le quotidien, en l'alimentant 
de ces petits gestes qui nous font nous sentir bien chaque jour. On commence par un sourire?»

A travers son site web, Danone se place comme une marque amie qui offre tous types de conseils notamment sur la santé, la nutrition, on trouve des recettes de cuisine, on y incite à la pratique d'un sport, etc. On y trouve même une section dédiée aux femmes qui sont considérées comme les chefs de familles. Des conseils simples mais aussi l'organisation hebdomadaire d'échanges avec des experts de la santé et des conférences sur le bonheur menées par de prestigieux philosophes et psychiatres espagnols.

Danone encourage aussi le sentiment de sympathie en nous familiarisant avec l'entreprise par le biais de diverses stratégies. En plus des classiques concours et promotions dont la campagne est faite, il est intéressant de noter la volonté de faire partager l'histoire même de la marque et la mise en vente d'objets vintage, transformant ainsi en pièces de collections d'anciens modèles de la marque. Une autre initiative originale consiste à établir des liens de camaraderie entre les différents publics de l'entreprise : les clients, les éleveurs et les fabricants du yaourt. C'est dans cet esprit qu'a vu le jour l'espace Danone à Barcelone, dans lequel est développé le concept d'expérience de marque, une stratégie qui vise à renforcer le lien émotionnel entre la marque et les clients. Il s'agit d'un restaurant, arborant la couleur blanche caractéristique de Danone, qui propose des menus sains ainsi qu'une yaourterie où l'on peut faire de nombreuses combinaisons au moment même de la commande et où l'on peut déguster des yaourts glacés de différents parfums, etc. Une autre stratégie se centre sur les fournisseurs, c'est-à-dire les éleveurs. En plus de vidéos qui présentent la journée de travail de ces derniers, le public est invité à aller visiter les granges et un concours à été mis en place pour gagner un week-end à la campagne en famille.

La sympathie et la proximité que génèrent ces actions se voient multipliées par les possibilités de diffusion qu'offrent les réseaux sociaux. C'est sur Facebook qu'il est possible de participer à une ambitieuse campagne de solidarité : chaque usager peut, s'il le souhaite, envoyer une photo de lui souriant avec un nez de clown et ainsi obtenir la visite d'un Docteur Sourire à un enfant hospitalisé. Il s'agit d'un projet de collaboration entre Danone et la Fondation Theodora, dont l'activité consiste en l'accompagnement des enfants hospitalisés.

Enfin en octobre 2013 a été menée une opération de street marketing au travers de laquelle une des principales stations du métro madrilène s'est transformée en un supermarché virtuel. Les murs de la station ont été recouverts d'affiches représentant des rayons remplis de produits Danone, chacun d'eux étant munis d'un code QR qui pouvaient être scannés par les téléphones mobiles et ainsi obtenir des bons de réductions. Une équipe de personnel de Danone, le « Commando des Economies » a été présente toute la journée afin de répondre aux éventuelles interrogations dans la «Station des Economies", selon le nom qui lui fut donné. Cette initiative a atteint 10000 personnes.

37 En fin de compte « chez Danone nous avons toujours voulu être aux côtés des familles, et aujourd'hui plus que jamais nous voulons continuer à l'être vu le contexte actuel (...) les petits gestes qui nous font esquisser un sourire et nous font sentir bien aident à ce que notre quotidien, et celui de ceux qui nous entourent, s'améliore. L'optimisme peut se transmettre et une attitude positive est fondamentale.» explique Carolina Clemente, Brand Manager chez Danone. ${ }^{10}$ 


\section{Conclusion} aliments, comme les yaourts Danone, ont transformé le discours publicitaire. Partant de la persuasion sur ses bénéfices pour la santé, le message publicitaire incite maintenant à l'expression d'émotions positives. Le consommateur connaît très bien la marque, ainsi que de nombreuses autres marques de la concurrence. Le besoin d'outils de communication efficaces dans un contexte concurrentiel amène au choix de messages publicitaires positifs. Ceux-ci exercent, d'une manière particulière un rôle stimulant sur le client, dans une époque marquée par de graves difficultés économiques. Le sourire représente le bien-être, l'absence de soucis, c'est le principal indicateur du bonheur, son langage est universel, il se comprend sans paroles.

Au-delà de l'émotion, la rationalité scientifique confirme les effets bénéfiques du sourire. Il nous met de bonne humeur et aide à vivre mieux et plus longtemps. Il a aussi des conséquences sociales puisqu'il se propage et répand la bonne humeur et la santé aux autres. C'est pour cela que Danone incite à sourire, à partager le sourire dans la vie de tous les jours, en famille, avec les fournisseurs, les enfants malades, et sur les réseaux sociaux. Sourire parce que Danone est à tes côtés. On est passé de l'utilisation de la publicité comme un outil de persuasion, au développement de relations publiques visant à établir des liens entre les différents publics de l'entreprise, et ce au travers de l'expression des émotions.

\section{NOTES}

1. Gerd Gigerenzer, Decisiones instintivas: la inteligencia del inconsciente, Barcelona, Ariel, 2008, p. 12.

2. Joan Costa, Imagen corporativa en el siglo XXI, Buenos Aires, La Crujía Editores, 2006.

3. Paul Capriotti, Planificación Estratégica de la Imagen Corporativa, Barcelona, Ariel, 1999.

4. Carl Iver Hovland, Irving Lester Janis, Harold H. Kelley, Communication and persuasion: psychological studies of opinion change, New Haven, Yale University Press, 1953.

5. William J. McGuire, «An information-processing model of advertising effectiveness », In H.L. David \& A.J. Silk (eds), Behavioral and Management Science in Marketing, New York: Ronald, 1978.

6. Robert B. Zajonc, R. B., "Feeling and thinking. Preferences need no inferences", American Psychologist, 1980, 35(2), 151-175.

7. Gordon H. Bower, "Mood and memory", American Psychologist, Vol 36(2), Feb 1981, 129-148.

8. Morris B. Holbrook, Elizabeth C., "The experiential aspects of consumption: consumer fantasies, feelings and fun ", Journal of Consumer Research, 1982, 9, 132-140.

9. URL : http://granadaempresas.es/la-nueva-publicidad-emocional/noticias/4885.html, Consulté le 7.11.2013.

10. URL : http://www.prnoticias.com/index.php/periodismo/1129-tendenciasprmarketing/20120537-danone-se-enfrenta-con-ternura-a-la-crisis-en-su-ultimo-spot, Consulté le 7.11.2013. 


\section{RÉSUMÉS}

Danone est l'une des entreprises les plus importantes dans la production de yaourts et de produits laitiers. Sa dernière campagne de communication lancée en mars 2013, en Espagne, est fondée sur le lien entre alimentation et bonheur. Sous le slogan "Danone alimente le sourire ", celle-ci tente de rapprocher l'image de marque avec le bonheur et les bons moments de la vie manifestés par le sourire. Tous les spots publicitaires abordent le thème du sourire et son rôle bienfaisant dans la vie quotidienne. Ce message corporatif s'adresse à un large public et aux femmes en particulier en tant que décideurs de l'alimentation et de la composition des courses familiales. C'est autour de ce lien entre alimentation et bonheur que s'est construit le discours de la marque: les styles de vie sains, les bienfaits du sourire, la promotion de week-ends à la campagne, la vie en famille... pour attirer les clients habituels de la marque. Un discours marqué par les valeurs d'authenticité, de proximité et de santé. L'objectif de cet article est de montrer le lien étroit entre alimentation, vie saine et bonheur mis en exergue par la campagne publicitaire du leader du secteur des produits laitiers. 